\title{
Neutropenia During Tocilizumab Treatment Is Not Associated with Infection Risk in Systemic or Polyarticular-course Juvenile Idiopathic Arthritis
}

\author{
Manuela Pardeo, Jianmei Wang, Nicolino Ruperto (D, Ekaterina Alexeeva, Vyacheslav Chasnyk, \\ Rayfel Schneider, Gerd Horneff, Hans-Iko Huppertz, Kirsten Minden, Karen Onel, \\ Lawrence Zemel, Alan Martin, Isabelle Koné-Paut, Antigoni Siamopoulou-Mavridou, \\ Clovis A. Silva, Benjamin Porter-Brown, Kamal N. Bharucha, Hermine I. Brunner, \\ and Fabrizio De Benedetti, for the Paediatric Rheumatology International Trials Organisation \\ (PRINTO) and the Pediatric Rheumatology Collaborative Study Group (PRCSG)
}

ABSTRACT. Objective. To determine whether neutropenia is associated with increased risk for infection in patients with systemic juvenile idiopathic arthritis (sJIA) and polyarticular-course juvenile idiopathic arthritis (pcJIA) treated with tocilizumab (TCZ).

Methods. Data up to Week 104 from 2 phase III trials of intravenous TCZ in sJIA ( $\mathrm{n}=112$; ClinicalTrials.gov, NCT00642460) and pcJIA $(n=188$; ClinicalTrials.gov, NCT00988221) were pooled. Worst common toxicity criteria grade and lowest observed absolute neutrophil count (ANC) were identified for each patient. Associations between patient characteristics and lowest observed ANC were tested using univariate regression analysis. Infection and serious infection rates per 100 patient-years (PY) in periods associated with grades $1 / 2$ and $3 / 4$ neutrophil counts were compared with rates associated with normal neutrophil counts.

Results. ANC decreased to grade $\geq 3$ in $25.0 \%$ and $5.9 \%$ of sJIA and pcJIA patients, respectively, and decreases were transient. Young age $(\mathrm{p}=0.047)$ and methotrexate use $(\mathrm{p}=0.012)$ were positively associated with neutropenia in patients with sJIA but not in patients with pcJIA. The rate of serious infections in patients with sJIA (10.9/100 PY; 95\% CI 6.8-16.5) tended to be higher than in patients with pcJIA (5.2/100 PY; 95\% CI 3-8.5). No increase in rates of serious or nonserious infections was observed during periods of neutropenia in either trial.

Conclusion. Patients with JIA treated with TCZ experienced transient neutropenia that was not associated with an increased number of infections. (First Release April 15 2019; J Rheumatol 2019;46:1117-26; doi:10.3899/jrheum.180795)

Key Indexing Terms:

BIOLOGICAL THERAPY

JUVENILE IDIOPATHIC ARTHRITIS

INFECTION

NEUTROPHILS

From the Institute for Research and Health Care (IRCCS) Ospedale Pediatrico Bambino Gesù, Division of Rheumatology, Rome, Italy; Roche Products Ltd., Welwyn Garden City, UK; IRCCS Istituto Giannina Gaslini, Clinica Pediatrica e Reumatologia, PRINTO, Genoa, Italy; Federal State Autonomous Institution National Medical Research Center of Children's Health of the Ministry of Health of the Russian Federation, Department of Rheumatology, and Federal State Autonomous Educational Institution of Higher Education I.M. Sechenov, First Moscow State Medical University of the Ministry of Health of the Russian Federation, Department of Pediatrics and Pediatric Rheumatology, Moscow; Saint-Petersburg State Pediatric Medical University, Department of Hospital Pediatrics, St. Petersburg, Russia; Hospital for Sick Children, University of Toronto, Department of Pediatrics, Division of Rheumatology, Toronto, Ontario, Canada; Asklepios Klinik Sankt Augustin, Centre for General Pediatrics and Neonatology, Sankt Augustin, and University Hospital of Cologne, Cologne; Prof. Hess Children's Hospital and Pediatric Intensive Care Medicine, Bremen; German Rheumatism Research Centre Berlin, and Charité University Medicine, Department of Rheumatology and Clinical Immunology, Berlin, Germany; Hospital for Special Surgery, Division of Pediatric Rheumatology, New York, New York; Connecticut Children's Medical Center, Pediatric Rheumatology, Hartford, Connecticut; Tulsa Bone and Joint Associates, Tulsa, Oklahoma; Genentech, South San
Francisco, California; University of Cincinnati, Cincinnati Children's Hospital Medical Center, Division of Rheumatology, Department of Pediatrics, PRCSG Coordinating Center, Cincinnati, Ohio, USA; National Referral Centre of Auto-Inflammatory Diseases, CEREMAIA, Centre Hospitalier Universitaire (CHU) de Bicere, Department of Pediatric Rheumatology, AP-HP, University of Paris Sud, Le Kremlin Bicêtre, France; University Hospital of Ioannina, Unit of Paediatric

Rheumatology, Paediatric Clinic, Ioannina, Greece; Children's Institute, Hospital das Clinicas, Faculdade de Medicina, Universidade de Sao Paulo (HCFMUSP), Pediatric Rheumatology Unit, Sao Paulo, Brazil.

The study was sponsored by F. Hoffmann-La Roche Ltd., which manufactures tocilizumab. Support for third-party writing assistance was provided by Sara Duggan, PhD, of ApotheCom and was funded by F. Hoffmann-La Roche Ltd. J. Wang is an employee of F. Hoffmann-La Roche Ltd. N. Ruperto is a consultant/speaker for F. Hoffmann-La Roche Ltd. E. Alexeeva is a consultant/speaker for F. Hoffmann-La Roche Ltd. $K$. Minden is a consultant/speaker or receives honoraria from F. Hoffmann-La Roche Ltd. I. Koné-Paut is a consultant to F. Hoffmann-La Roche Ltd. B. Porter-Brown is an employee of and owns stock in F. Hoffmann-La Roche Ltd. K. Bharucha owns stock in F. Hoffmann-La Roche Ltd. H.I. Brunner is a consultant/speaker or receives honoraria from $F$. Hoffmann-La Roche Ltd. F. De Benedetti is a

Personal non-commercial use only. The Journal of Rheumatology Copyright $\subset$ (2019. All rights reserved. 
Interleukin 6 (IL-6) is a cytokine implicated in many aspects of inflammation ${ }^{1,2}$, making it an attractive target for treating a variety of chronic inflammatory diseases. Clinical trials have demonstrated the efficacy and safety of the anti-IL-6 receptor- $\alpha$ antibody tocilizumab (TCZ) in treating rheumatoid arthritis (RA) ${ }^{3,4,5,6,7,8}$, giant cell arteritis ${ }^{9}$, systemic juvenile idiopathic arthritis (sJIA), and polyarticular-course juvenile idiopathic arthritis (pcJIA) ${ }^{10,11}$.

Neutropenia was reported in all clinical trials performed with TCZ. In RA trials, $4.8 \%$ of patients reported common toxicity criteria (CTC) $)^{12}$ grade 3 [absolute neutrophil count (ANC) $0.5-<1 \times 10^{9} / 1$ ] neutropenia, and $0.7 \%$ reported grade 4 (ANC $\left.<0.5 \times 10^{9} / 1\right)$ neutropenia ${ }^{13}$. Grade 3 or grade 4 neutropenia was also observed in phase III clinical trials in children with sJIA and with pcJIA; frequencies were $25.0 \%$ and $5.9 \%$ of patients, respectively, during 2 years of treatment with $\mathrm{TCZ}^{14,15}$. In patients with RA, neutropenia associated with TCZ administration was usually transient $(90 \%$ of patients with grade 3 or 4 neutropenia experienced it on a single visit, 2 consecutive visits, or nonconsecutive visits) and did not lead to treatment discontinuation ${ }^{16}$. However, the

consultant/speaker or receives honoraria from F. Hoffmann-La Roche Ltd. M. Pardeo, MD, IRCCS Ospedale Pediatrico Bambino Gesù, Division of Rheumatology; J. Wang, PhD, Roche Products Ltd.; N. Ruperto, MD, MPH, IRCCS Istituto Giannina Gaslini, Clinica Pediatrica e Reumatologia, PRINTO; E. Alexeeva, MD, PhD, Professor, Federal State Autonomous Institution National Medical Research Center of Children's Health of the Ministry of Health of the Russian Federation, Department of Rheumatology, and Federal State Autonomous Educational Institution of Higher Education I.M. Sechenov, First Moscow State Medical University of the Ministry of Health of the Russian Federation, Department of Pediatrics and Pediatric Rheumatology; V. Chasnyk, MD, Professor, Saint-Petersburg State Pediatric Medical University, Department of Hospital Pediatrics; R. Schneider, MBBCh, Hospital for Sick Children, University of Toronto, Department of Pediatrics, Division of Rheumatology; G. Horneff, MD, Professor, Asklepios Klinik Sankt Augustin, Centre for General Pediatrics and Neonatology, Sankt Augustin, and University Hospital of Cologne; H.I. Huppertz, MD, Professor, Prof. Hess Children's Hospital and Pediatric Intensive Care Medicine; K. Minden, MD, German Rheumatism Research Centre Berlin, and Charité University Medicine, Department of Rheumatology and Clinical Immunology; K. Onel, MD, Professor, Hospital for Special Surgery, Division of Pediatric Rheumatology; L. Zemel, MD, Connecticut Children's Medical Center, Pediatric Rheumatology; A. Martin, MD, Tulsa Bone and Joint Associates; I. Koné-Paut, MD, National Referral Centre of Auto-Inflammatory Diseases, CEREMAIA, CHU de Bicere, Department of Pediatric Rheumatology, AP-HP, University of Paris Sud; A. Siamopoulou-Mavridou, MD, Professor, University Hospital of Ioannina, Unit of Paediatric Rheumatology, Paediatric Clinic; C.A. Silva, MD, PhD, Associate Professor, Children's Institute, HCFMUSP, Pediatric Rheumatology Unit; B. Porter-Brown, MBBS, DPM, MFPM, Roche Products Ltd.; K.N. Bharucha, PhD, MD, Genentech (affiliation at time of the study); H.I. Brunner, MD, MSc, University of Cincinnati, Cincinnati Children's Hospital Medical Center, Division of Rheumatology, Department of Pediatrics, PRCSG Coordinating Center; F. De Benedetti, $M D, P h D$, IRCCS Ospedale Pediatrico Bambino Gesù, Division of Rheumatology. M. Pardeo and J. Wang contributed equally to the study.

Address correspondence to Dr. F. De Benedetti, IRCCS Ospedale

Pediatrico Bambino Gesù, Piazza S. Onofrio 4, 00165 Rome, Italy. E-mail: fabrizio.debenedetti@opbg.net

Full Release Article. For details see Reprints and Permissions at jrheum.org

Accepted for publication December 19, 2018. potential relationship between decreased neutrophil count and increased risk for infection remains a cause of concern, particularly in children treated with TCZ.

Treatment with sarilumab, another anti-IL-6R monoclonal antibody, leads to similar effects on neutrophils ${ }^{17}$. The mechanism leading to reduced neutrophil counts during IL-6R blockade remains unknown. Although IL-6 has been shown to have a role in the stimulation of hematopoietic progenitor stem cells ${ }^{18}$, a direct role on neutropoiesis has not been clearly demonstrated. IL-6 knockout (KO) mice do not show neutropenia; however, crossing the severely neutropenic granulocyte-colony-stimulating factor (G-CSF) receptor KO mice with IL-6 KO mice led to further decreases in neutrophil counts, suggesting that IL-6 might play a role in this extreme situation ${ }^{19}$

Some studies have suggested several potential mechanisms, including increased neutrophil apoptosis and reduced neutrophil survival, increased margination and/or migration/trafficking of neutrophils in peripheral tissue, and decreases in other proinflammatory cytokines, including those that have an effect on neutropoiesis ${ }^{20,21,22}$.

We performed a secondary analysis of the data from the 2 pivotal phase III clinical trials of TCZ in patients with SJIA $^{10}$ and pcJIA ${ }^{11}$ to investigate variables possibly associated with reduced neutrophil counts and to evaluate their relationship to the development of serious and nonserious infections.

\section{MATERIALS AND METHODS}

Patients and study designs. TENDER (ClinicalTrials.gov, NCT00642460) was a 5-year, phase III study that assessed the efficacy and safety of intravenous TCZ in 112 patients with sJIA aged 2 to 17 years. The active disease was defined by criteria of the International League of Associations for Rheumatology (ILAR) ${ }^{23}$ and had lasted $\geq 6$ months. Weight-adjusted TCZ doses were administered: patients were randomly assigned to receive TCZ ( $12 \mathrm{mg} / \mathrm{kg}$ for body weight $<30 \mathrm{~kg}$ or $8 \mathrm{mg} / \mathrm{kg}$ for body weight $\geq 30 \mathrm{~kg}$ ) or placebo every 2 weeks. Patients who had baseline white blood cell counts $<5.0 \times 10^{9} / 1$ and neutrophil counts $<2.5 \times 10^{9} / 1$ were excluded from enrolling. Additional details are included in the Supplementary Material (available with the online version of this article) and have been published ${ }^{10}$.

CHERISH (ClinicalTrials.gov, NCT00988221) was a 2-year, 3-part, randomized, double-blind, placebo-controlled withdrawal study of the efficacy and safety of TCZ in 188 patients aged 2 to 17 years. They had rheumatoid factor (RF)-positive or RF-negative pcJIA or extended oligoarticular JIA with polyarticular course for $\geq 6$ months before study entry, according to ILAR criteria ${ }^{23}$. Part 1 was a 16 -week, active-treatment period during which patients who weighed $\geq 30 \mathrm{~kg}$ received TCZ $8 \mathrm{mg} / \mathrm{kg}$ and patients who weighed $<30 \mathrm{~kg}$ were randomly assigned 1:1 to receive TCZ $8 \mathrm{mg} / \mathrm{kg}$ or $10 \mathrm{mg} / \mathrm{kg}$ every 4 weeks for 4 doses. Patients who achieved at least a JIA American College of Rheumatology 30 response at Week 16 could enter part 2 (24-week, double-blind, placebo-controlled withdrawal period) and were randomly assigned to receive placebo or to continue TCZ at the same dose received in part 1. Part 3 was an additional 64-week, open-label TCZ extension period. Patients with baseline white blood cell counts $<5.0 \times 10^{9} / 1$ and those with neutrophil counts $<2.5 \times 10^{9} / 1$ were excluded. Additional details of the CHERISH trial are published ${ }^{11}$. Data up to Week 104 from patients with sJIA in the TENDER trial and patients with pcJIA in the CHERISH trial were pooled for the current analysis.

Both studies were conducted in accordance with the Declaration of Helsinki, Good Clinical Practice guidelines, and local requirements, and both

Personal non-commercial use only. The Journal of Rheumatology Copyright @ 2019 . All rights reserved. 
studies were approved by the institutions' Research Ethics Boards (the approval number for the principal trial site 165340 for CHERISH was $73 / 2009$ and for TENDER, was $220 \mathrm{VA} / \mathrm{cm}$ ). Parents or guardians provided written informed consent and patients provided written informed assent.

Study assessments. Blood cell counts were monitored at each study visit in both studies. According to the study protocols, TCZ administration was delayed if patients had neutrophil counts lower than $1 \times 10^{9} / 1$. Infection adverse events (AE) were identified and reported as per the Medical Dictionary for Regulatory Activities system organ class code of Infections and Infestations. TCZ serum concentrations were measured using an ELISA (Endo Drug Development) ${ }^{24}$.

Statistical analysis. Analysis included the all-exposure safety population (all patients who received $\geq 1$ dose of TCZ and who underwent $\geq 1$ safety assessment after TCZ administration). Placebo treatment periods for patients who received placebo in part 1 of TENDER and part 2 of CHERISH, respectively, were excluded from the analysis, unless stated otherwise. Worst CTC neutropenia grade and lowest neutrophil counts $\left(10^{9} / 1\right)$ were graded for each patient using the National Cancer Institute Common Terminology Criteria for Adverse Events, version 3.012. The grading system classifies severity of neutropenia as follows: grade 0 , normal; grade $1,<$ lower limit of normal $(\mathrm{LLN})$ to $1.5 \times 10^{9} / 1$; grade $2,<1.5$ to $1.0 \times 10^{9} / 1$; grade $3,<1.0$ to $0.5 \times$ $10^{9} / 1$; and grade $4,<0.5 \times 10^{9} / 1$. The LLN used in the definition of grade 1 low neutrophil count was age- and sex-specific for this pediatric population.

Associations between patient characteristics at baseline, concomitant treatments, and patients' TCZ exposure estimated by the average $\mathrm{C}_{\text {trough }}$ at the time of the lowest observed neutrophil counts were determined using univariate linear regression analysis. Variables that might have affected neutrophil counts were preselected for inclusion in the regression analysis. All variables analyzed were included in the results. Rates of infection and serious infection (Supplementary Material, available with the online version of this article) associated with neutropenia episodes were compared with corresponding rates associated with periods of normal neutrophil counts.

\section{RESULTS}

Baseline demographics and disease characteristics. Baseline demographics and disease characteristics of all patients are shown in Table 1. At baseline, oral glucocorticoids (GC) were used by $91 \%(102 / 112)$ of patients with sJIA at a mean (SD) dose of $0.3(0.18) \mathrm{mg} / \mathrm{kg} /$ day; $69 \%(77 / 112)$ of these patients received methotrexate [MTX; 13.7 (SD 8.4) $\mathrm{mg} / \mathrm{m}^{2} /$ week]. Among the patients with pcJIA, 46\% (86/188) were treated with $\mathrm{GC}$ at baseline $[0.13(\mathrm{SD} 0.05) \mathrm{mg} / \mathrm{kg} /$ day]; $79 \%$
(148/188) received MTX [13.0 (SD 5.8) $\mathrm{mg} / \mathrm{m}^{2} /$ week]. Differences in demographics and characteristics between the groups (body weight $\geq 30 \mathrm{~kg}$ vs body weight $<30 \mathrm{~kg}$ ), including age, weight, and disease duration, were as expected according to the body weight-based dosing regimens.

Neutrophil count during treatment with TCZ. At baseline, the median (interquartile range; IQR) ANC among patients with sJIA was $8.13(6.52-12.89) \times 10^{9} / 1$. About $50 \%$ of patients had ANC exceeding their age-specific upper limit of normal range, and no patients had ANC below normal at baseline. Median ANC decreased from $8.13 \times 10^{9} / 1$ at baseline to 3.93 $\times 10^{9} / 1$ within 2 weeks of the initiation of TCZ treatment, then stabilized and remained within the normal range up to Week 104 in the group of patients with sJIA (Figure 1A). Notably, $44.6 \%(n=50)$ of patients with sJIA had normal ANC or grade 1 neutropenia throughout the study. Grade 2 neutropenia occurred in 34 patients $(30.4 \%)$, grade 3 in 26 patients (23.2\%), and grade 4 in 2 patients (1.8\%; Table 2). A numerically higher proportion of patients weighing $<30$ $\mathrm{kg}[36 \%(21 / 58)]$ than patients weighing $\geq 30 \mathrm{~kg}[13.0 \%$ (7/54)] had CTC grade 3/4 neutropenia. In total, it occurred in 28 patients ( 2 had grade 4 ); in 14 of those patients, it occurred once during the trial. One patient experienced 3 episodes of grade 3/4 neutropenia that lasted up to 14 days each. Grade 3 neutropenia occurred once during an episode of macrophage activation syndrome (MAS); the other 4 episodes of potential or definite MAS observed in TENDER did not occur with low ANC.

In patients with pcJIA, the median (IQR) baseline ANC was $4.8(3.6-6.0) \times 10^{9} / 1$ for all patients. About $7 \%$ of these patients had ANC exceeding the upper limit of normal, and no patients had neutrophil counts below normal at baseline. The median ANC decreased after TCZ initiation and stabilized after Week 16; similar patterns were observed in patients with pcJIA who received placebo in part 2 of the study (Figure 1B). Overall, 70.7\% $(\mathrm{n}=133)$ of patients had normal or minimally decreased ANC (grade 1) throughout

Table 1. Baseline demographics and disease characteristics of all patients.

\begin{tabular}{|c|c|c|c|c|c|c|}
\hline \multirow[t]{2}{*}{ Characteristics } & \multicolumn{3}{|c|}{ sJIA } & \multicolumn{3}{|c|}{ pcJIA } \\
\hline & All, $N=112$ & $\mathrm{BW}<30 \mathrm{~kg}, \mathrm{n}=58$ & $\mathrm{BW} \geq 30 \mathrm{~kg}, \mathrm{n}=54$ & All, $N=188$ & $\mathrm{BW}<30 \mathrm{~kg}, \mathrm{n}=69$ & $\mathrm{BW} \geq 30 \mathrm{~kg}, \mathrm{n}=119$ \\
\hline Mean age, yrs (SD) & $9.7(4.6)$ & $6.4(3.2)$ & $13.2(2.9)$ & $11.0(4.0)$ & $7.2(2.9)$ & $13.1(2.8)$ \\
\hline Mean body weight, $\mathrm{kg}(\mathrm{SD})$ & $33.8(19.6)$ & $20.1(5.9)$ & $48.5(18.5)$ & $39.6(17.3)$ & $21.5(5.5)$ & $50.0(12.6)$ \\
\hline Mean disease duration, yrs (SD) & $5.2(4.1)$ & $3.7(3.1)$ & $6.7(4.5)$ & $4.2(3.7)$ & $3.5(2.46)$ & $4.7(4.2)$ \\
\hline Mean baseline neutrophil count, $10^{9} / 1$ (SD) & $10.7(6.9)$ & $11.4(7.4)$ & $9.9(6.3)$ & $5.4(3.2)$ & $5.6(2.0)$ & $5.2(3.7)$ \\
\hline Baseline MTX use, $n(\%)$ & $77(69)$ & $46(79)$ & $31(57)$ & $148(79)$ & $59(86)$ & $89(75)$ \\
\hline Mean baseline MTX dose, ${ }^{\mathrm{a}} \mathrm{mg} /$ week (SD) & $13.7(8.4)$ & $13.1(10.1)$ & $14.6(4.8)$ & $13.0(5.8)$ & $15.1(8.1)$ & $11.6(2.7)$ \\
\hline
\end{tabular}

Patients with sJIA weighing $<30 \mathrm{~kg}$ received TCZ $12 \mathrm{mg} / \mathrm{kg}$ every 2 weeks, and those weighing $\geq 30 \mathrm{~kg}$ received TCZ $8 \mathrm{mg} / \mathrm{kg}$ every 2 weeks. Patients with pcJIA weighing $<30 \mathrm{~kg}$ received TCZ $8 \mathrm{mg} / \mathrm{kg}$ or TCZ $10 \mathrm{mg} / \mathrm{kg}$ every 4 weeks, and those weighing $\geq 30 \mathrm{~kg}$ received TCZ $8 \mathrm{mg} / \mathrm{kg}$ every $4 \mathrm{weeks}$. ${ }^{\text {a }} \mathrm{Mean}$ dose based on patients who were taking GC or MTX only. BW: body weight; GC: glucocorticoids; MTX: methotrexate; pcJIA: polyarticular-course juvenile idiopathic arthritis; sJIA: systemic juvenile idiopathic arthritis; TCZ: tocilizumab.

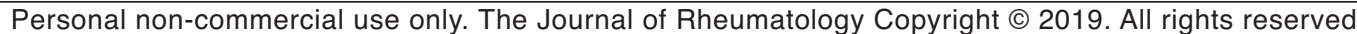




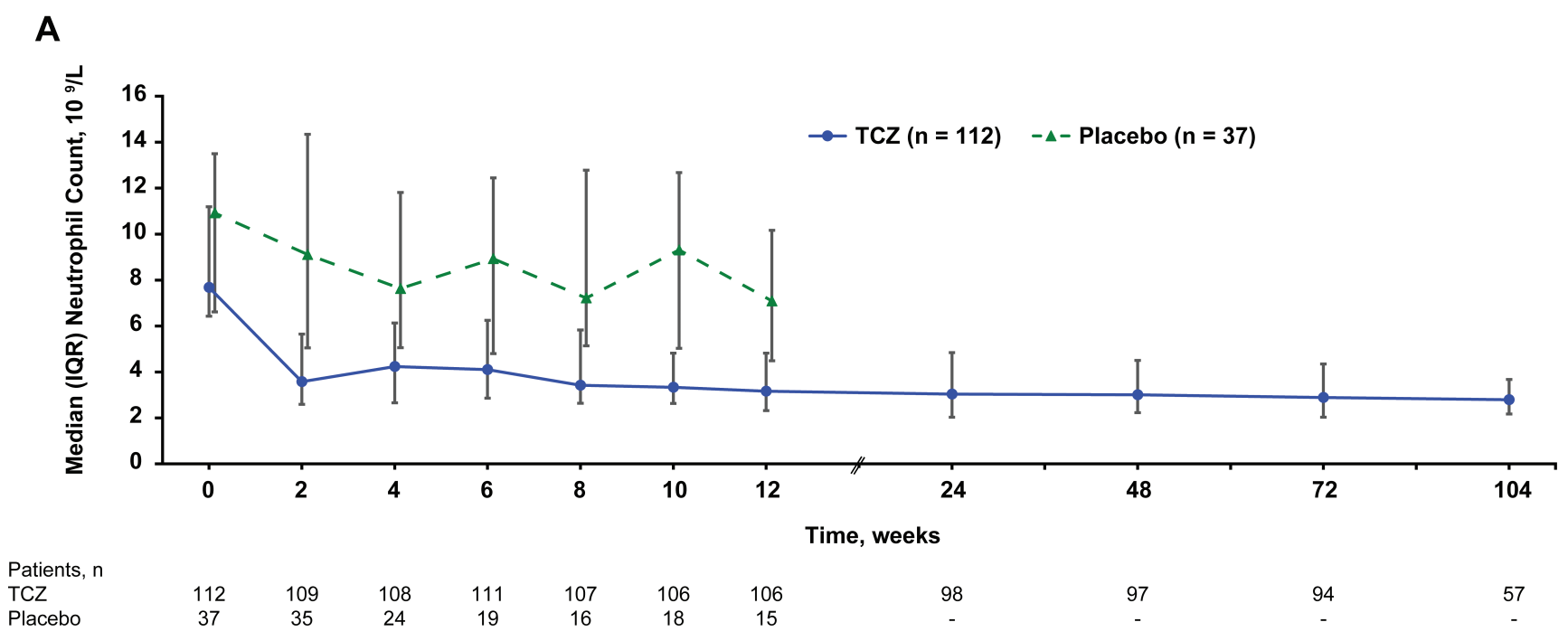

B

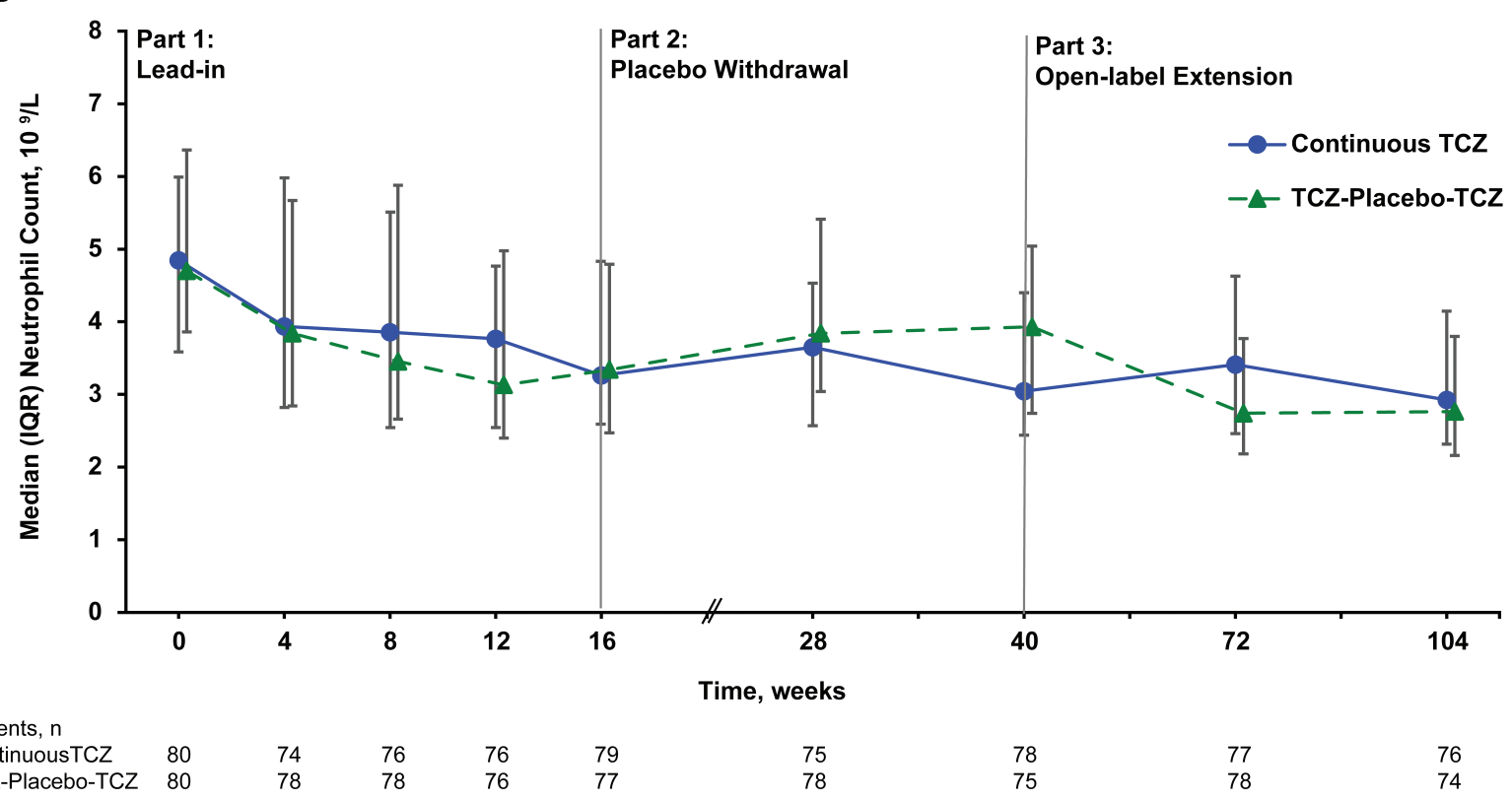

Figure 1. A. Median (IQR) neutrophil count in patients with sJIA treated with TCZ for up to 2 years. B. Median (IQR) neutrophil count in patients with pcJIA stratified by part 2 randomized treatment. IQR: interquartile range; pcJIA: polyarticular-course juvenile idiopathic arthritis; sJIA: systemic juvenile idiopathic arthritis; TCZ: tocilizumab.

the study; CTC grade 2 or 3 neutropenia was reported in 44 $(23.4 \%)$ or $11(5.9 \%)$ patients, respectively. None of the patients with pcJIA experienced grade 4 neutropenia (Table 2 ). A greater proportion of patients with pcJIA weighing $<30$ $\mathrm{kg}$ and receiving TCZ $10 \mathrm{mg} / \mathrm{kg}$ [10.1\% (7/69)] experienced episodes of grade 3 neutrophil counts than patients weighing $\geq 30 \mathrm{~kg}$ and receiving TCZ $8 \mathrm{mg} / \mathrm{kg}$ [3.4\% (4/119)].

Among these 11 patients with CTC grade 3 neutropenia, 8 experienced neutropenia once, 2 experienced nonconsec- utive episodes of neutropenia, and 1 experienced grade 3 neutropenia at 3 consecutive timepoints over 60 days.

Factors associated with the development of neutropenia during TCZ treatment. Univariate linear regression analysis was used to examine the associations among baseline age, $\mathrm{GC}$, and MTX use and dose, and TCZ $\mathrm{C}_{\text {trough }}$ at the time of the lowest ANC (Figures 2A and 2B).

In patients with sJIA, though neither GC use (yes vs no; absolute or weight-adjusted dose; regression coefficient 0.35 , 
Table 2. Worst CTC grade of neutropenia during the first 2 years of TCZ treatment in sJIA and pcJIA patients by BW group.

\begin{tabular}{|c|c|c|c|c|c|c|}
\hline \multirow[t]{2}{*}{ CTC Grade } & \multicolumn{3}{|c|}{ sJIA } & \multicolumn{3}{|c|}{ pcJIA } \\
\hline & All, $N=112$ & $\mathrm{BW}<30 \mathrm{~kg}, \mathrm{n}=58$ & $\mathrm{BW} \geq 30 \mathrm{~kg}, \mathrm{n}=54$ & All, $N=188$ & $\mathrm{BW}<30 \mathrm{~kg}, \mathrm{n}=69$ & $\mathrm{BW} \geq 30 \mathrm{~kg}, \mathrm{n}=119$ \\
\hline Grade $0-1, \mathrm{n}(\%), 1.5 \times 10^{9} / 1$ to normal & 50 (44.6) & $21(36.2)$ & $29(53.7)$ & 133 (70.7) & 49 (71.0) & 84 (70.6) \\
\hline Grade $3, \mathrm{n}(\%), 0.5$ to $<1.0 \times 10^{9} / 1$ & $26(23.2)$ & $19(32.8)$ & $7(13.0)$ & $11(5.9)$ & $7(10.1)$ & $4(3.4)$ \\
\hline Grade $4, \mathrm{n}(\%),<0.5 \times 10^{9} / 1$ & $2(1.8)$ & $2(3.4)$ & 0 & 0 & 0 & 0 \\
\hline
\end{tabular}

Patients with sJIA weighing $<30 \mathrm{~kg}$ received TCZ $12 \mathrm{mg} / \mathrm{kg}$ every 2 weeks, and those weighing $\geq 30 \mathrm{~kg}$ received TCZ $8 \mathrm{mg} / \mathrm{kg}$ every 2 weeks. Patients with pcJIA weighing $<30 \mathrm{~kg}$ received TCZ $8 \mathrm{mg} / \mathrm{kg}$ or TCZ $10 \mathrm{mg} / \mathrm{kg}$ every 4 weeks, and those weighing $\geq 30 \mathrm{~kg}$ received TCZ $8 \mathrm{mg} / \mathrm{kg}$ every 4 weeks. BW: body weight; CTC: common toxicity criteria; pcJIA: polyarticular-course juvenile idiopathic arthritis; sJIA: systemic juvenile idiopathic arthritis; TCZ: tocilizumab.

A

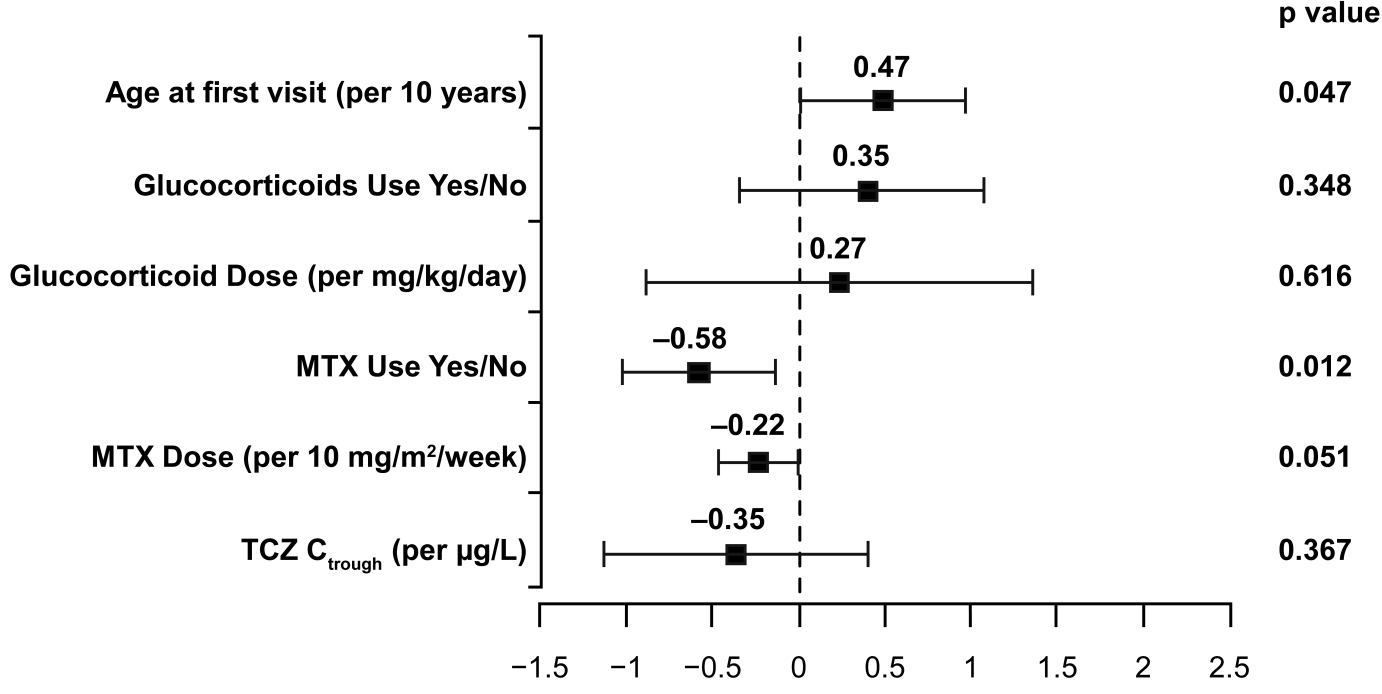

Difference $(95 \% \mathrm{Cl})$ in lowest neutrophil count, $10 \% \mathrm{~L}$

B

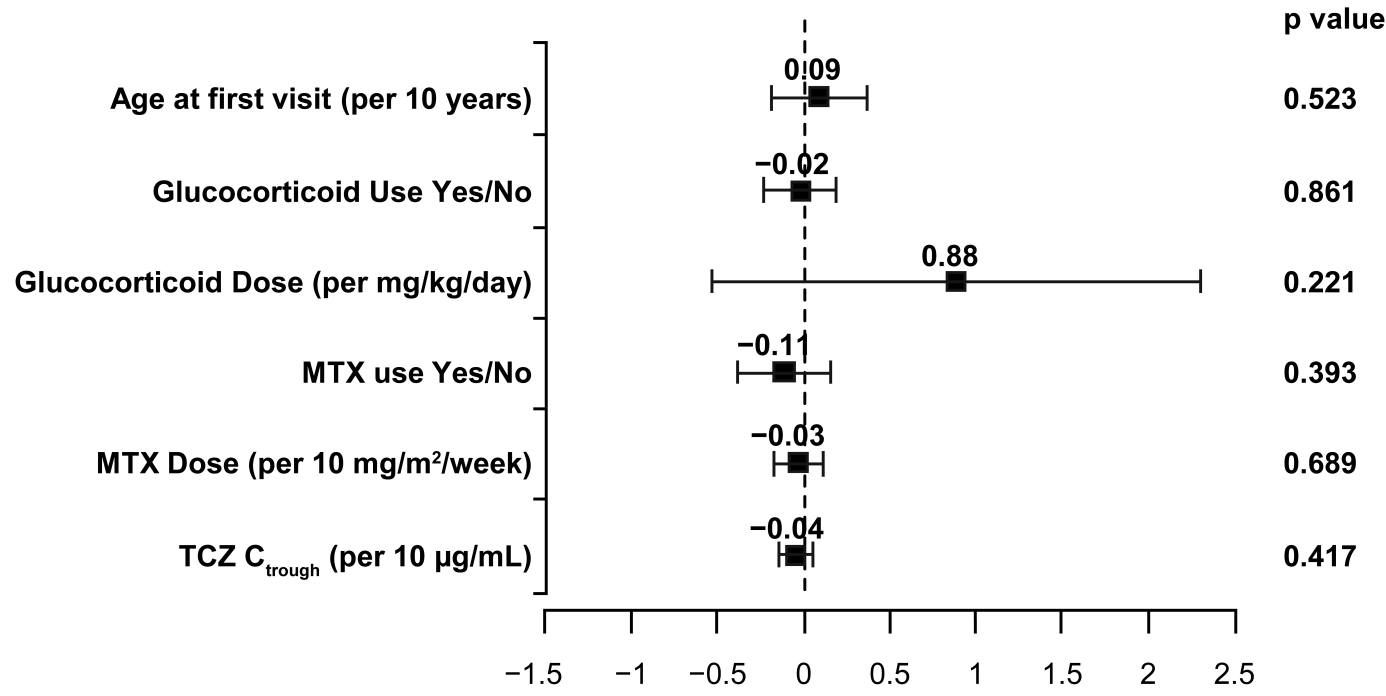

Difference $(95 \% \mathrm{CI})$ in lowest neutrophil count, $10 \% \mathrm{~L}$

Figure 2. Association between baseline and pharmacokinetic variables with neutrophil counts based on univariate regression analysis in (A) patients with sJIA and (B) patients with pcJIA. Differences are regression coefficients against each variable. For example, 0.47 for age (A) means that older age (per $10 \mathrm{yrs}$ ) corresponds to an increase in the lowest neutrophil count by $0.47 \times 10^{9} / 1$. $\mathrm{C}_{\text {trough }}$ : within-subject average trough TCZ concentration; MTX: methotrexate; pcJIA: polyarticular-course juvenile idiopathic arthritis; sJIA: systemic juvenile idiopathic arthritis; TCZ: tocilizumab.

Personal non-commercial use only. The Journal of Rheumatology Copyright @ 2019 . All rights reserved. 
$95 \% \mathrm{CI}-0.39$ to $1.10 ; \mathrm{p}=0.348$ or $0.27,95 \% \mathrm{CI}-0.81$ to $1.35 ; \mathrm{p}=0.616$ ) nor $\mathrm{TCZ} \mathrm{C}_{\text {trough }}$ (regression coefficient $-0.35,95 \% \mathrm{CI}-1.11$ to $0.42 ; \mathrm{p}=0.367$ ) was associated with the lowest ANC, younger age showed a weak, though significant, positive association (regression coefficient $0.47,95 \%$ CI $0.01-0.93 ; p=0.047$ ). Additionally, background MTX use was significantly associated with the lowest ANC (regression coefficient $-0.58,95 \% \mathrm{CI}-1.02$ to $-0.13 ; \mathrm{p}=0.012)$, and there was a trend toward association with the MTX dose $\left(\mathrm{mg} / \mathrm{m}^{2} /\right.$ week) and the minimum ANC (regression coefficient $-0.22,95 \% \mathrm{CI}-0.45$ to $0.00 ; \mathrm{p}=0.051$; Figure $2 \mathrm{~A}$ ). None of these associations found in patients with sJIA were observed in patients with pcJIA (Figure 2B). Neutropenia was not associated with exposure to TCZ as measured by TCZ trough levels (regression coefficient, $-0.04,95 \% \mathrm{CI}-0.14$ to 0.06 ; $\mathrm{p}=0.417$; Figure 2B).

Infections and serious infections during treatment with TCZ. Among patients with sJIA, 13 infection AE (243.7/100 PY) were reported for $29.7 \%$ of patients during placebo exposure compared with 46 infection AE (267.6/100 PY) reported for $45.3 \%$ of patients during TCZ exposure. By Week 104, 102 of $112(91.1 \%)$ patients reported a total of 570 infection AE (282.1/100 PY). Overall, the most frequently reported infections were nasopharyngitis and upper respiratory tract infections. In patients with sJIA treated with TCZ, 22 serious infection AE developed in 20 patients (17.9\%), corresponding to a rate of $10.9 / 100 \mathrm{PY}$. There were 4 events each $(2.0 / 100 \mathrm{PY})$ of gastroenteritis and varicella, 3 events $(1.5 / 100 \mathrm{PY})$ of pneumonia, and 2 events (1.0/100 PY) of herpes zoster. All cases of varicella or herpes zoster were treated with acyclovir, and all resolved without sequelae. All other serious infections were single events and also resolved without sequelae.

Among patients with pcJIA, 465 infection AE (151.4/100 PY) were reported for 134 of 188 patients (71.3\%) by Week 104. The most frequently reported infections were nasopharyngitis (27.4/100 PY), upper respiratory tract infection (15.3/100 PY), and pharyngitis (11.7/100 PY). Overall, 16 serious infections were reported in 14 patients with pcJIA (7.4\%), corresponding to a rate of 5.2/100 PY during TCZ treatment. There were 4 events (1.3/100 PY) of pneumonia and 2 events each (0.7/100 PY) of bronchitis, cellulitis, and varicella. One case of varicella was untreated and 1 was treated with acyclovir; both resolved without sequelae. All other serious infections were single events and also resolved during the study period.

Relationship between infection and neutropenia. Among patients with sJIA, the rate of infection AE was comparable between periods of normal (276.5/100 PY), grade $1 / 2$ (226.7/100 PY), and grade 3/4 neutropenia (292.5/100 PY; Figure $3 \mathrm{~A})$. Infections occurring around periods of grade $1 / 2$ neutropenia included nasopharyngitis (15 events), upper respiratory tract infection ( 9 events), pharyngitis (4 events), and impetigo and urinary tract infection (3 events each). The remaining reported infections were also single events. Infections occurring around periods of grade $3 / 4$ neutropenia included upper respiratory tract infection (5 events), nasopharyngitis (3 events), gastroenteritis and conjunctivitis ( 2 events each), and pneumonia, rhinitis, viral infection, and subcutaneous abscess ( 1 event each). No serious infections were reported around episodes of grade 3 or 4 neutropenia, but 2 serious infections were reported with grade $1 / 2$ neutropenia (8.7/100 PY), which is similar to the rate of infection AE (11.5/100 PY) observed during periods of normal neutrophil counts (Figure 3B).

Among patients with pcJIA, rates of infection AE during periods of normal, grade $1 / 2$, and grade $3 / 4$ neutropenia were 147.8/100 PY, 176.6/100 PY, and 340/100 PY, respectively, with largely overlapping CI (Figure $3 \mathrm{C}$ ). Infections occurring around periods of grade $1 / 2$ neutropenia included nasopharyngitis (6 events), upper respiratory tract infection (6 events), pharyngitis (4 events), respiratory tract infection (3 events), and gastroenteritis, influenza, pediculosis, localized infection, and rhinitis ( 2 events each). All other infections were single events. Infections with grade $3 / 4$ neutropenia included upper respiratory tract infection and influenza (2 events each) and mumps, tracheitis, and nasopharyngitis (1 event each). No serious infections were reported around episodes of neutropenia of any grade. All 16 serious infections occurred during periods of normal neutrophil counts and $\geq 30$ days from neutrophil counts below the lower limit of normal (Figure 3D).

\section{DISCUSSION}

Neutropenia was reported in the 2 pivotal trials of TCZ in patients with JIA, with grade $\geq 3$ events occurring in 19 of 112 patients with sJIA (17\%) after 52 weeks ${ }^{10}$ and in 7 of 188 patients with pcJIA $(3.7 \%)$ after 40 weeks ${ }^{11}$. This secondary analysis included data for up to 2 years of TCZ treatment in each trial, identified potential risk factors for the development of neutropenia, and investigated whether there was a temporal association between neutropenia and the occurrence of infections. In this analysis, grade $\geq 3$ neutropenia was observed in $25.0 \%$ of the patients with sJIA and in $5.9 \%$ of the patients with pcJIA. Risk factors for neutropenia included young age and MTX use in patients with sJIA but not in patients with pcJIA. Rates of infection (serious or nonserious) were similar during times of normal or near-normal ANC (grade 1 and grade 2 neutropenia) and periods of more pronounced reduction of neutrophil counts (grade 3 or 4 neutropenia) in patients with sJIA and in those with pcJIA. Grade $\geq 3$ neutropenia has been reported in clinical trials of TCZ in patients with RA. In a longterm pooled analysis of the pivotal phase 3 and 4 clinical trials of TCZ in RA (16,204.8 PY of TCZ exposure), grade 3 neutropenia occurred in $5.4 \%$ of patients and grade 4 neutropenia occurred in $<1 \%$ of patients ${ }^{18}$. Hence, the frequency of neutropenia in patients with pcJIA (5.9\%) reported here is

Personal non-commercial use only. The Journal of Rheumatology Copyright @ 2019 . All rights reserved. 
A

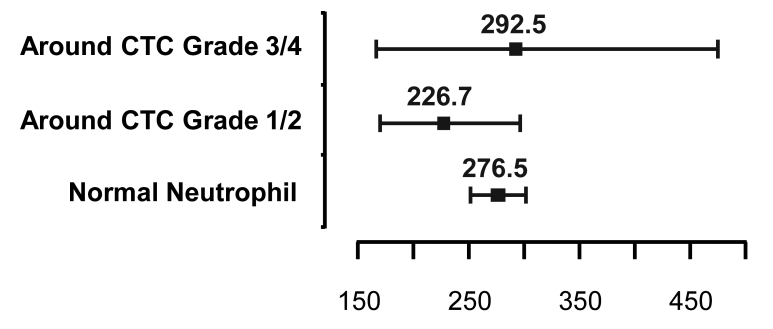

Rates of Infection AEs /100 PY

B

Around CTC Grade 3/4
Around CTC Grade 1/2
Normal Neutrophil

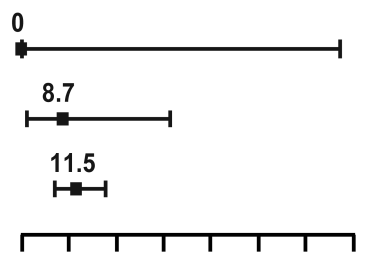

$\begin{array}{llllllll}0 & 10 & 20 & 30 & 40 & 50 & 60 & 70\end{array}$

Rates of Serious Infection AEs /100 PY
Total PY \# Events

$5.5 \quad 16$

$22.9 \quad 52$

$173.6 \quad 480$
Total PY \# Events

$\begin{array}{rr}5.5 & 0 \\ 22.9 & 2 \\ 173.6 & 20\end{array}$

C

Around CTC Grade 3/4
Around CTC Grade 1/2
Normal Neutrophil

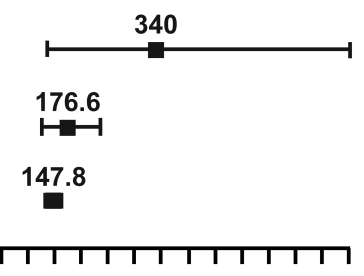

$\begin{array}{llllll}0 & 100 & 250 & 400 & 550 & 700\end{array}$

Rates of Infection AEs /100 PY
Total PY \# Events

$\begin{array}{rr}2.1 & 7 \\ 25.5 & 45 \\ 279.5 & 413\end{array}$

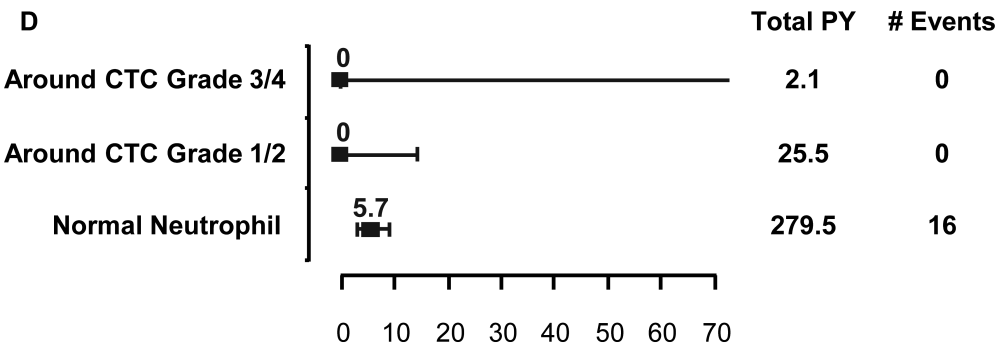

Rates of Serious Infection AEs /100 PY

Figure 3. Rates of (A) infection and (B) serious infection during \pm 15 days of normal neutrophil count, grade 1 or 2 neutrophil count, and grade 3 or 4 neutrophil count in patients with sJIA treated with tocilizumab. Rates of (C) infection and (D) serious infection during \pm 30 days of normal neutrophil count, grade 1 or 2 neutrophil count, and grade 3 or 4 neutrophil count in patients with pcJIA treated with tocilizumab. AE: adverse event; PY: patient-years; CTC: common toxicity criteria; pcJIA: polyarticular-course juvenile idiopathic arthritis; sJIA: systemic juvenile idiopathic arthritis.

comparable to that reported in patients with RA. As might be expected, neutropenia was more common among patients with RA who received TCZ $8 \mathrm{mg} / \mathrm{kg}$ than those who received
TCZ $4 \mathrm{mg} / \mathrm{kg}$ per month ${ }^{4,6}$. Together with the data from patients with sJIA reported here, this suggests that higher TCZ exposure is associated with more frequent neutro-

$$
\text { Personal non-commercial use only. The Journal of Rheumatology Copyright @) 2019. All rights reserved. }
$$


penia ${ }^{4,6}$. However, we did not observe a direct relationship between TCZ mean serum concentrations and ANC in patients with sJIA or in those with pcJIA. This suggests that other factors may also contribute to the development of decreases in neutrophil count. Although our study lacked the power for more informative multivariate analysis, it is worth noting that, based on univariate analysis, MTX background therapy was a risk factor for neutropenia in patients with sJIA. However, it was not a risk factor in patients with pcJIA and has not been reported as a risk factor in patients with RA treated with $\mathrm{TCZ}^{3}$. Moreover, in a metaanalysis of Japanese patients with RA treated with TCZ monotherapy, the incidence of grade 3 neutropenia $(6 \%)^{25}$ was not different from that reported in global trials in which TCZ was used in combination with $\mathrm{MTX}^{16}$. It is possible that other disease-related factors are involved in the development of neutropenia or that the effect of MTX on neutrophil counts is evident only with higher doses of TCZ, such as those administered to patients with sJIA. Given the limited, if any, efficacy of MTX in sJIA ${ }^{26}$, holding TCZ is recommended in patients with neutrophil counts $<1.0 \times 10^{9} / 1$, and withdrawing MTX or dose reduction might be considered.

The use of GC is associated with neutrophilia because they inhibit neutrophil apoptosis in humans ${ }^{27}$. Our current analysis showed that GC use (yes or no) and weight-adjusted GC dose were not significantly associated with lowest neutrophil count. This finding suggests that GC treatment does not mask neutropenia in patients treated with TCZ. It should be noted that a potential limitation of our current study is the reduction in GC dose over the course of the study as disease improved.

In both sJIA and pcJIA, children in the lower weight category had a higher frequency of grade 3 neutropenia; analysis of the relationship between lowest neutrophil count and several variables showed a significant association only for age and only in patients with sJIA. The prevalence of neutropenia is higher in younger subjects. In a US-based epidemiological study, neutropenia was observed in $3.7 \%$ of children aged 3 to 5 years but in only $1.5 \%$ of adolescents aged 15 to 17 years and in $0.72 \%$ of adults ${ }^{28}$.

Our data support the finding that the rate of serious infections in children with sJIA treated with TCZ (10.9/100 PY, 95\% CI 6.8-16.5) tends to be higher than in children with pcJIA treated with TCZ (5.2/100 PY, 95\% CI 3-8.5) and still higher than reported in adult patients with RA treated with TCZ with or without MTX (4.4/100 PY, 95\% CI 4.1-4.8) $)^{16}$. This result is consistent with observations of other treatments previously reported. For example, in patients with sJIA treated with anakinra, the rate of infections was about $6 / 100$ $\mathrm{PY}$ in a multicenter retrospective case series of 46 patients ${ }^{29}$ and 26/100 PY in a randomized, double-blind, placebo-controlled study in 22 patients ${ }^{30}$. Similarly, in a large US report on infections in patients with JIA, Beukelman, et al ${ }^{31}$ showed a risk for infections requiring hospital admission in anakinra users (primarily patients with sJIA) at a rate of 8.4/100 PY. In agreement with these findings, preliminary results from the Pharmachild Registry showed that the risk for infection was significantly increased in patients with sJIA (about 2 -fold higher) than in patients with other JIA subtypes ${ }^{32}$. Indeed, the rate of serious infection reported in trials of patients with pcJIA treated with tumor necrosis factor inhibitors (TNFi) ranges from 2/100 PY to 4/100 PY $\mathrm{PY}^{33,34}$. These numbers are consistent with a large study in patients with JIA observed for about 13,000 PY that showed an overall rate of 2.8/100 PY for bacterial infections that necessitated hospital admission, which was 2-fold higher than in the control population (patients with attention-deficit hyperactivity disorder) and 3.5-fold higher in patients who received TNFi with or without MTX ${ }^{35}$.

The data reported here support the idea that susceptibility to infections is a feature of JIA and the conclusion that inflammation/autoimmunity predisposes children to infections; this predisposition may be amplified by individual treatments. Further, GC, particularly at high doses, are known to increase the risk for infections in adults with $\mathrm{RA}^{36,37,38,39}$ and in children with JIA ${ }^{32,35}$. Patients with sJIA are more commonly treated with higher doses of GC than patients with pcJIA; this was also true for the populations analyzed in our study.

In the sJIA and the pcJIA trials, we found a numerically higher rate of infections around grade 3 or 4 neutropenia than around normal or grade 1 or 2 neutropenia; however, serious infections were not more commonly reported during periods of grade 3 and 4 neutropenia than around normal or grade 1 and 2 neutropenia. It is worth noting that we evaluated the occurrence of infections during grade $\geq 3$ neutropenia over a duration of 5.5 years in patients with sJIA and 2.1 years in patients with pcJIA. In an observational study in patients with severe congenital neutropenia, the rate of serious infection was 232/100 PY before the administration of G-CSF and 38/100 PY after diagnosis and the administration of G-CSF ${ }^{40}$.

A number of hypotheses may be consistent with the lack of an association between decreased ANC during TCZ treatment and the occurrence of infections. It is possible that blockade of IL-6 may modulate the circulating pool of neutrophils by shifting them from the circulating to the marginated pools ${ }^{21,41}$, or by increasing their transit time through the bone marrow, or both ${ }^{21,42}$. Moreover, after TCZ administration, neutrophils are fully functional and show no impairment in their antibacterial functions ${ }^{40}$.

Our findings indicate that neutropenia in patients with sJIA and patients with pcJIA who were treated with TCZ was transient and was not associated with the development of infections and serious infections.

\section{ACKNOWLEDGMENT}

The authors thank the patients who were enrolled in this study and their families. They extend special thanks to all the trial investigators for their energy and commitment.

Personal non-commercial use only. The Journal of Rheumatology Copyright @ 2019 . All rights reserved. 


\section{ONLINE SUPPLEMENT}

Supplementary material accompanies the online version of this article.

\section{REFERENCES}

1. Smolen JS, Maini RN. Interleukin-6: a new therapeutic target. Arthritis Res Ther 2006;8:S5.

2. Wright HL, Cross AL, Edwards SW, Moots RJ. Effects of IL-6 and IL-6 blockade on neutrophil function in vitro and in vivo. Rheumatology 2014;53:1321-31.

3. Maini RN, Taylor PC, Szechinski J, Pavelka K, Broll J, Balint G, et al. Double-blind randomized controlled clinical trial of the interleukin-6 receptor antagonist, tocilizumab, in European patients with rheumatoid arthritis who had an incomplete response to methotrexate. Arthritis Rheum 2006;54:2817-29.

4. Smolen JS, Beaulieu A, Rubbert-Roth A, Ramos-Remus C, Rovensky J, Alecock E, et al. Effect of interleukin-6 receptor inhibition with tocilizumab in patients with rheumatoid arthritis (OPTION study): a double-blind, placebo-controlled, randomised trial. Lancet 2008;371:987-97.

5. Genovese MC, McKay JD, Nasonov EL, Mysler EF, da Silva NA, Alecock E, et al. Interleukin-6 receptor inhibition with tocilizumab reduces disease activity in rheumatoid arthritis with inadequate response to disease-modifying antirheumatic drugs: the tocilizumab in combination with traditional disease-modifying antirheumatic drug therapy study. Arthritis Rheum 2008;58:2968-80.

6. Emery P, Keystone E, Tony HP, Cantagrel A, van Vollenhoven R, Sanchez A, et al. IL-6 receptor inhibition with tocilizumab improves treatment outcomes in patients with rheumatoid arthritis refractory to anti-tumour necrosis factor biologicals: results from a 24-week multicentre randomised placebo-controlled trial. Ann Rheum Dis 2008;67:1516-23

7. Jones G, Sebba A, Gu J, Lowenstein MB, Calvo A, Gomez-Reino JJ, et al. Comparison of tocilizumab monotherapy versus methotrexate monotherapy in patients with moderate to severe rheumatoid arthritis: the AMBITION study. Ann Rheum Dis 2010;69:88-96.

8. Kremer JM, Blanco R, Brzosko S, Burgos-Vargas R, Halland AM, Vernon E, et al. Tocilizumab inhibits structural joint damage in rheumatoid arthritis patients with inadequate responses to methotrexate: results from the double-blind treatment phase of a randomized placebo-controlled trial of tocilizumab safety and prevention of structural joint damage at one year. Arthritis Rheum 2011;63:609-21.

9. Stone JH, Tuckwell K, Dimonaco S, Klearman M, Aringer M, Blockmans D, et al. Trial of tocilizumab in giant-cell arteritis. N Engl J Med 2017;377:317-28.

10. De Benedetti F, Brunner HI, Ruperto N, Kenwright A, Inmaculada C, Cuttica R, et al. Randomized trial of tocilizumab in systemic juvenile idiopathic arthritis. N Engl J Med 2012;367:2385-95.

11. Brunner HI, Ruperto N, Zuber Z, Keane C, Harari O, Kenwright A, et al. Efficacy and safety of tocilizumab in patients with polyarticular-course juvenile idiopathic arthritis: results from a phase 3, randomised, double-blind withdrawal trial. Ann Rheum Dis 2014;74:1110-7.

12. Cancer Therapy Evaluation Program. Common Terminology Criteria for Adverse Events v3.0 (CTCAE). [Internet. Accessed March 5, 2019.] Available from: ctep.cancer.gov/ protocoldevelopment/electronic_applications/docs/ctcaev3.pdf.

13. Genovese MC, Rubbert-Roth A, Smolen JS, Kremer J, Khraishi M, Gomez-Reino J, et al. Longterm safety and efficacy of tocilizumab in patients with rheumatoid arthritis: a cumulative analysis of up to 4.6 years of exposure. J Rheumatol 2013;40:768-80.

14. De Benedetti F, Rubio-Perez N, Goodman S, Job-Delandre C, Joos $\mathrm{R}$, Kone-Paut I, et al. Neutropenia with tocilizumab treatment is not associated with increased infection risk in patients with polyarticular-course juvenile idiopathic arthritis. Arthritis Rheumatol 2014;66:S67-8.

15. De Benedetti F, Ruperto N, Baildam E, Burgos-Vargas R, Horneff $\mathrm{G}$, Iko Huppertz H, et al. Neutropenia with tocilizumab treatment is not associated with increased infection risk in patients with systemic juvenile idiopathic arthritis [abstract]. Arthritis Rheum 2013;65 Suppl 10:S114.

16. Moots RJ, Sebba A, Rigby W, Ostor A, Porter-Brown B, Donaldson F, et al. Effect of tocilizumab on neutrophils in adult patients with rheumatoid arthritis: pooled analysis of data from phase 3 and 4 clinical trials. Rheumatology 2017;56:541-9.

17. Huizinga TW, Fleischmann RM, Jasson M, Radin AR, van Adelsberg J, Fiore S, et al. Sarilumab, a fully human monoclonal antibody against IL-6Ralpha in patients with rheumatoid arthritis and an inadequate response to methotrexate: efficacy and safety results from the randomised SARIL-RA-MOBILITY Part A trial. Ann Rheum Dis 2014;73:1626-34.

18. Rennick D, Jackson J, Yang G, Wideman J, Lee F, Hudak S. Interleukin-6 interacts with interleukin-4 and other hematopoietic growth factors to selectively enhance the growth of megakaryocytic, erythroid, myeloid, and multipotential progenitor cells. Blood 1989;73:1828-35.

19. Liu F, Poursine-Laurent J, Wu HY, Link DC. Interleukin-6 and the granulocyte colony-stimulating factor receptor are major independent regulators of granulopoiesis in vivo but are not required for lineage commitment or terminal differentiation. Blood 1997;90:2583-90.

20. Gibiansky L, Frey N. Linking interleukin-6 receptor blockade with tocilizumab and its hematological effects using a modeling approach. J Pharmacokinet Pharmacodyn 2012;39:5-16.

21. Suwa T, Hogg JC, English D, van Eeden SF. Interleukin-6 induces demargination of intravascular neutrophils and shortens their transit in marrow. Am J Physiol Heart Circ Physiol 2000;279:H2954-60.

22. Lok LSC, Farahi N, Juss JK, Loutsios C, Solanki CK, Peters AM, et al. Effects of tocilizumab on neutrophil function and kinetics. Eur J Clin Invest 2017;47:736-45.

23. Petty RE, Southwood TR, Manners P, Baum J, Glass DN, Goldenberg $\mathrm{J}$, et al. International League of Associations for Rheumatology classification of juvenile idiopathic arthritis: second revision, Edmonton, 2001. J Rheumatol 2004;31:390-2.

24. Stubenrauch K, Wessels U, Birnboeck H, Ramirez F, Jahreis A, Schleypen J. Subset analysis of patients experiencing clinical events of a potentially immunogenic nature in the pivotal clinical trials of tocilizumab for rheumatoid arthritis: evaluation of an antidrug antibody ELISA using clinical adverse event-driven immunogenicity testing. Clin Ther 2010;32:1597-609.

25. Nishimoto N, Ito K, Takagi N. Safety and efficacy profiles of tocilizumab monotherapy in Japanese patients with rheumatoid arthritis: meta-analysis of six initial trials and five long-term extensions. Mod Rheumatol 2010;20:222-32.

26. Woo P, Southwood TR, Prieur A-M, Doré CJ, Grainger J, David J, et al. Randomized, placebo-controlled, crossover trial of low-dose oral methotrexate in children with extended oligoarticular or systemic arthritis. Arthritis Rheum 2000;43:1849-57.

27. Saffar AS, Ashdown H, Gounni AS. The molecular mechanisms of glucocorticoids-mediated neutrophil survival. Curr Drug Targets 2011;12:556-62.

28. Hsieh MM, Everhart JE, Byrd-Holt DD, Tisdale JF, Rodgers GP. Prevalence of neutropenia in the U.S. population: age, sex, smoking status, and ethnic differences. Ann Intern Med 2007;146:486-92.

29. Nigrovic PA, Mannion M, Prince FH, Zeft A, Rabinovich CE, van Rossum MA, et al. Anakinra as first-line disease-modifying therapy in systemic juvenile idiopathic arthritis: report of forty-six patients from an international multicenter series. Arthritis Rheum 2011;63:545-55.

Personal non-commercial use only. The Journal of Rheumatology Copyright (C) 2019. All rights reserved. 
30. Quartier P, Allantaz F, Cimaz R, Pillet P, Messiaen C, Bardin C, et al. A multicentre, randomised, double-blind, placebo-controlled trial with the interleukin-1 receptor antagonist anakinra in patients with systemic-onset juvenile idiopathic arthritis (ANAJIS trial). Ann Rheum Dis 2011;70:747-54.

31. Beukelman T, Xie F, Baddley JW, Chen L, Mannion ML, Saag KG, et al. The risk of hospitalized infection following initiation of biologic agents versus methotrexate in the treatment of juvenile idiopathic arthritis. Arthritis Res Ther 2016;18:210.

32. Giancane G, Swart J, Bovis F, Castagnola E, Groll A, Horneff G, et al. Risk of infections in juvenile idiopathic arthritis patients treated with biologic agents and/or methotrexate: results from Pharmachild Registry [abstract]. Arthritis Rheumatol 2016;68 Suppl 10:3116.

33. Lovell DJ, Ruperto N, Goodman S, Reiff A, Jung L, Jarosova K, et al. Adalimumab with or without methotrexate in juvenile rheumatoid arthritis. N Engl J Med 2008;359:810-20.

34. Lovell DJ, Reiff A, Jones OY, Schneider R, Nocton J, Stein LD, et al. Long-term safety and efficacy of etanercept in children with polyarticular-course juvenile rheumatoid arthritis. Arthritis Rheum 2006;54:1987-94.

35. Beukelman T, Xie F, Chen L, Baddley JW, Delzell E, Grijalva CG, et al. Rates of hospitalized bacterial infection associated with juvenile idiopathic arthritis and its treatment. Arthritis Rheum 2012;64:2773-80.
36. Doran MF, Crowson CS, Pond GR, O'Fallon WM, Gabriel SE Frequency of infection in patients with rheumatoid arthritis compared with controls: a population-based study. Arthritis Rheum 2002;46:2287-93.

37. Doran MF, Crowson CS, Pond GR, O'Fallon WM, Gabriel SE. Predictors of infection in rheumatoid arthritis. Arthritis Rheum 2002;46:2294-300.

38. Smitten AL, Choi HK, Hochberg MC, Suissa S, Simon TA, Testa MA, et al. The risk of hospitalized infection in patients with rheumatoid arthritis. J Rheumatol 2008;35:387-93.

39. Bernatsky S, Hudson M, Suissa S. Anti-rheumatic drug use and risk of serious infections in rheumatoid arthritis. Rheumatology 2007;46:1157-60.

40. Fioredda F, Calvillo M, Burlando O, Riccardi F, Caviglia I, Tucci F, et al. Infectious complications in children with severe congenital, autoimmune or idiopathic neutropenia: a retrospective study from the Italian Neutropenia Registry. Pediatr Infect Dis J 2013;32:410-2.

41. Hashizume M, Higuchi Y, Uchiyama Y, Mihara M. IL-6 plays an essential role in neutrophilia under inflammation. Cytokine 2011;54:92-9.

42. Suwa T, Hogg JC, Klut ME, Hards J, van Eeden SF. Interleukin-6 changes deformability of neutrophils and induces their sequestration in the lung. Am J Respir Crit Care Med 2001;163:970-6. 